Materials Sciences Division, Lawrence Berkeley National Laboratory, and

Department of Materials Science and Engineering

University of California at Berkeley

\title{
THE IDEAL STRENGTH OF IRON IN TENSION AND SHEAR
}

\section{D.M. Clatterbuck ${ }^{1}$, D.C. Chrzan ${ }^{1,2}$ and J.W. Morris, Jr. ${ }^{1,2}$.}

${ }^{1}$ Materials Sciences Division, Lawrence Berkeley National Laboratory, and

Department of Materials Science and Engineering,

University of California, Berkeley, CA 94720

${ }^{2}$ Corresponding Author: Tel: (510) 486-5994; Fax: (510) 486-4023

E-mail address: dcchrzan@socrates.berkeley.edu (D.C.Chrzan)

${ }^{2}$ Corresponding Author: Tel: (510) 486-6482; Fax: (510) 486-4023

E-mail address: jwmorris@uclink4.berkeley.edu (J.W. Morris, Jr.)

March 2003

Accepted for Publication:

Acta Materialia, Vol. 51; pp.2271-2283

This work was supported by the Director, Office of Science, Office of Basic Energy Sciences, of the Department of Energy under contract No. DE-AC03-76SF00098. 


\title{
The ideal strength of iron in tension and shear
}

\author{
D. M. Clatterbuck, D. C. Chrzan, J. W. Morris Jr.** \\ Center for Advanced Materials, Lawrence Berkeley National Lab and Department of Materials \\ Science and Engineering, University of California, Berkeley, CA 94720 \\ **Electronic Address: jwmorris@uclink4.berkeley.edu
}

\begin{abstract}
The ideal strength of a material is the stress at which the lattice itself becomes unstable and, hence, sets a firm upper bound on the mechanical strength the material can have. The present paper includes an ab-initio calculation of the ideal shear strength of Fe. It is, to our knowledge, the first such computation for any ferromagnetic material. The paper also elaborates on our earlier calculation of the ideal tensile strength of $\mathrm{Fe}$ by studying the effects of strains which break the tetragonal symmetry. The strengths were calculated using the Projector Augmented Wave Method within the framework of density functional theory and the generalized gradient approximation. In $<001>$ tension the ideal strength is determined by an elastic instability of the ferromagnetic phase along the "Bain" strain path from bcc to fcc. An $<001>$ tensile strain also leads to instability with respect to transformation into a face centered orthorhombic structure, and to various magnetic instabilities. However, these are encountered at larger strains and, thus, do not affect the ideal strength. We also investigated the ideal shear strength of bcc iron in two prominent shear systems, $<111>\{112\}$ and $<111>\{110\}$. In both shear systems the ideal strength is determined by the body centered tetragonal structure that defines a nearby saddle point on the energy surface. The ideal shear strengths are thus very similar, though they are not identical since the two shears follow slightly different strain paths from bcc to bct. We investigated the magnetic instabilities encountered during $<111>\{112\}$ shear. These instabilities do not appear until the strain is significantly greater than the instability strain of the ferromagnetic crystal. Hence while Fe exhibits some novel effects due to magnetism, they do not affect the ideal strength, which is determined by the same elastic instabilities that determine the strengths of most other bcc metals.
\end{abstract}


Keywords: Iron; Mechanical properties; Ideal strength; Ab-initio electron theory; Magnetic structure

\section{INTRODUCTION}

The ideal mechanical strength of a material is the stress required to force deformation or fracture by the elastic instability of its crystal lattice. The ideal strength has been the source of scientific inquiry for over 80 years [1]. The simple models for the ideal strength developed by Frenkel [2] and Orowon [3] have proven to be convenient means of understanding this topic. However, recent advances in the capabilities of computing machines have made it possible to study the ideal strength quantitatively and in greater detail using ab-initio electronic structure calculations [4-9].

While crystalline materials are normally deformed or broken by defects that become active at stresses well below the ideal strength, the ideal strength is interesting and important for several reasons. First, the ideal strength is one of the few mechanical properties of materials that can be computed from first principles. It is an inherent property of the crystal lattice and, thus, offers insight into the connection between the intrinsic bonding and symmetry of the crystal and the mechanical properties it shows. In fact, many of the familiar aspects of the mechanical behavior of crystalline solids, such as the $\{100\}$ cleavage of bcc crystals, the cleavage resistance of fcc materials, and the common slip systems of fcc and bcc are inherent features of behavior at the limit of strength [10]. Second, the ideal strength is an inherent upper bound on the strength. The ideal strength is useful to know since it sets clear limits on the attainable mechanical properties. For example, the ideal strength suggests a practical upper bound on the yield strength of ductile steel, and suggests why $\mathrm{Ni}$ additions may promote ductile fracture in high strength steel [11]. Third, as is becoming increasingly evident, the ideal strength is actually approached in a number of practical situations, such as the nano-indentation of materials with low defect densities [12].

While the ideal strength is defined by elastic instability, its precise definition is somewhat subtle. Of course, the ideal strength depends on the geometry of the load; the strength in tension depends on the direction of the tension, the strength in shear on the plane and direction of shear, the strength under multiaxial loading on the geometry of the multiaxial load. But the load that 
induces instability in an actual test may also depend on the response of the loading mechanism $[13,14]$. In this case the measured value of the limiting strength is not necessarily a material property. Nonetheless, there is a well-defined inherent value of the ideal strength for any load geometry [15]. It can be found from Gibbs' condition of internal stability: it is necessary (though not sufficient) for mechanical stability that the material be stable with respect to internal rearrangements that leave its boundaries fixed [15]. This condition is satisfied for a strained solid when every possible incremental strain increases the free energy. The tensor that governs the change in free energy on incremental strain from a strained state is the "Wallace tensor" [16], and the condition of stability is that all eigenvalues of the Wallace tensor be positive. A test sample in a loading device may fail at a load below the ideal strength defined by the first vanishing eigenvalue of the Wallace tensor, but it must fail when this strength is reached.

The elements of the Wallace tensor incorporate the elastic constants of the crystal in its current state of strain. While it is possible to compute the elastic constants for every step along a hypothetical strain path in an ab-initio study of elastic stability, it is tedious to do so. And it is usually unnecessary. We are ordinarily interested in the ideal strength for some simple example of the applied stress, such as uniaxial tension or simple shear. In these cases the ideal strength is ordinarily the maximum stress along the "relaxed" loading path in which all stresses orthogonal to the applied stress are zero. The possible exceptions involve instabilities that are orthogonal to the loading direction, such as a hypothetical shear instability that terminates deformation in uniaxial tension. It follows that the ideal stress can, ordinarily, be found directly by straining the material with the load of interest up to the maximum stress, while periodically applying low symmetry distortions to test for orthogonal instabilities. This procedure is well suited for $a b$ initio calculations.

Ab-initio computations of the ideal strengths of bcc metals (W, Mo, $\mathrm{Nb}, \mathrm{Fe}$ ) have been published by several investigators, for example [9,17-19]. The results show that symmetry plays a dominant role in determining the ideal strength. The energy of the crystal is a function of the six independent components of the strain and is, hence, a 6-dimensional hypersurface in a 7dimensional space. The local minima and the saddle points on this hypersurface almost always correspond to structures with high symmetry. The configurations of greatest interest are the 
saddle points in the energy surface that surround the initial state. The ideal strength is determined by the steepest slope encountered along the path from the initial state to the first saddle point. Thus each elastic instability is associated with a saddle point structure. Four possible saddle point structures have been identified in previous studies of the the ideal strengths of bcc metals: the fcc structure, the simple cubic (sc) structure, a body centered tetragonal (bct), and a base centered orthorhombic structure $[17,19,20]$. For example, relaxed shear of a bcc metal in the "easy" $<111>$ direction on the $\{112\}$ or $\{110\}$ plane generates a stress-free bct structure [19]. Because the $<111>\{112\}$ shear system is not symmetric in the sign of the shear direction, it is also possible to move from a bcc structure to a base centered orthorhombic structure by shearing in the "hard" direction. Uniaxial tension in the $<111>$ direction takes a bcc structure to a sc structure [17,20], while $<001>$ tension moves from bcc to either fcc (the Bain transformation [7]) or the same stress-free bct structure encountered in shear [19]. The competition between the two possible paths that can be reached by $<001>$ tension has the result that, while most bcc crystals (Mo, W) are governed by the instability associated with the fcc structure, some $(\mathrm{Nb})$ become unstable with respect to evolution toward the bct saddle point [19].

The ideal strength of $\mathrm{Fe}$ is both scientifically interesting and computationally challenging. A good part of the scientific interest and the computational challenge are due to its complex magnetic behavior. Scientifically, the magnetism of Fe has the consequence that fcc $\mathrm{Fe}$ is metastable, while it is an unstable phase in the other common bcc transition metals [18]. Fcc Fe is stabilized by a magnetic transition to a complex antiferromagnetic state, which has the consequence that the fcc phase is a minimum rather than a saddle point on the strain-energy surface of Fe. Because the ideal strength is ordinarily determined by the nature and location of the saddle point structures, one might expect that Fe would behave very differently than other bcc metals. As we shall see, however, it does not, and the reasons why it does not are scientifically interesting.

Computationally, the non-local magnetic interactions in Fe have the consequence that ab-initio computational methods that employ the local density approximation cannot be used. In fact, the local density approximation predicts that the ground state of Fe should be a non-magnetic close packed structure rather than a bcc ferromagnet. The use of the generalized gradient 
approximation has been shown to correct this problem [21]. However these results suggest that the energy differences between various phases are due to subtle differences in their charge density and charge density gradients. Full-potential Linearized Augmented Plane Wave (FLAPW) calculations which make no further approximations beyond the GGA (assuming convergence of the basis set, charge density representation and BZ integration) are probably the most reliable. Unfortunately, in order to efficiently relax the stresses orthogonal to the applied stress, the stresses on the unit cell must be directly computable, and there are no implementations of the FLAPW method known to us which can compute stresses. For the current work we have chosen to use the Projector Augmented Wave (PAW) Method, originally developed by Blöchl [22]. As we shall show below, this method offers both convenience and acceptable accuracy.

\section{COMPUTATIONAL METHODS}

We computed the total energy and the Hellman-Feynman stresses within the framework of density functional theory using the Projector Augmented Wave (PAW) Method [22]. We specifically used the PAW Method implementation within the Vienna Ab-initio Simulation Package (VASP) [23-25]. To implement this method we took the local density part of the exchange correlation energy from the Perdew and Zunger [26] parameterization of the Ceperly and Alder [27] exchange correlation functional, and adopted the Vosko-Wilk-Nusair (VWN) [28] spin interpolation of the local correlation energy. We used the gradient corrections of Perdew and Wang (PW92) [29-30] with the exception that the correlation part of the GGA was interpolated from the non-magnetic to the magnetic regime using the standard interpolation instead of the VWN interpolation formula. We used a scalar relativistic treatment for the $3 \mathrm{~d}$ and $4 \mathrm{~s}$ valence states, while treating the lower energy levels with the frozen core approximation. The plane-wave expansion had an energy cut-off of $25 \mathrm{Ry}$. We employed the modified tetrahedral method of Blöchl [31] for integration in the Brillouin zone, with the number of k-points dependent on the structure and number of atoms per unit cell in order to converge the energy to within $0.1 \mathrm{mRy}$ and the Hellman-Feynman stresses to within $0.1 \mathrm{GPa}$. In general a 17x17x17 Monkhorst Pack Grid was sufficient; however, some calculations were done with a more dense mesh of k-points. The stresses for several structures were also compared to calculations which used the integration scheme of Methfessel-Paxton [32] and were found to agree within 2\%. 
The ideal strength was computed for uniaxial stress in tension or shear. The lattice vectors were incrementally deformed in the direction of the imposed stress, and at each step the structure was relaxed until the stresses orthogonal to the applied stress vanished, as indicated by those components of the Hellman-Feynman stresses being less than $0.15 \mathrm{GPa}$ [8]. Because there is no unique measure of strain for a given finite deformation, we describe our deformations in terms of the engineering strain from the equilibrium structure. The initial set of lattice vectors $r^{\alpha}(\alpha=1,2,3)$ in an orthogonal coordinate system become the vectors $r^{\alpha}$ after homogeneous deformation by the transformation $r_{i}^{\alpha}=r_{i}^{\alpha}+D_{i j} r_{j}^{\alpha}$. From this transformation, we define the

strain to be $e_{i j}=\left(D_{i j}+D_{j i}\right) / 2$. As is customary, we redefine the shear strains to be $\gamma_{i j}=2 e_{i j}$ for $i \neq j$. While the engineering strain is convenient for describing the change in the lattice vectors from their original configuration, the Cauchy (true) stress cannot be calculated from the derivative of the free energy with respect to this strain measure. To compute the Cauchy stress we take the derivative of the free energy with respect to the incremental strain from a nearby reference state, yielding a stress that converges to the thermodynamic definition of the Cauchy stress in the limit of small incremental strain.

It should be noted that the ideal strengths determined from these calculations are for quasi-static deformation at $0 \mathrm{~K}$, and that other dynamic instabilities such as soft phonons may lower the ideal strength.

\section{RESULTS}

\section{A. Equilibrium structures}

We chose to compute the ideal strength with the Projector Augmented Wave (PAW) method because of its computational efficiency and its ability to treat lattice stress. To check the accuracy of the method, we computed the energy as a function of volume for several magnetic structures that had previously been calculated with the FLAPW method (see [18] for computational details). The magnetic structures included the following: bcc ferromagnet (FM), 
fcc ferromagnet (FM), fcc anti-ferromagnet (AFM), fcc non-magnetic (NM). The results are compared in Table 1.

In general the agreement between the two methods is good. In comparing the PAW and FLAPW methods we find that the equilibrium volumes of the various phases agree to within $1 \%$. The elastic constants of the bcc FM phase agree to within 3\% with the exception of $\mathrm{c}_{44}$ which has an discrepancy of $13 \%$. The agreement in the energy differences between the various magnetic phases is not quite as good with errors ranging from 3-16\%; however, these differences are probably of the same magnitude as the errors the generalized gradient approximation introduces as compared to the true density functional groundstate. In comparing the PAW potential used in this study with independent FLAPW calculations, Kresse and Joubert (1999) found that there was a $12 \%$ discrepancy in the energy difference between the fcc NM and bcc FM phases (using GGA), of the same order as the error reported here. We also note that the calculations used slightly different representations of the exchange correlation energy; the FLAPW calculations used the correlation energy and gradient expansions given in [29, 33]. However, both representations are roughly based on PW92.

Compared with experimental measurements of the bec phase at $4 \mathrm{~K}$, the PAW calculations predict a lattice parameter that is too small by $1 \%[34]$ and elastic constants which are generally about $10 \%$ too large [35] suggesting a slight over-binding (the only discrepancy being $\mathrm{c}_{44}$ which is $18 \%$ too small). From the computed elastic constants, the relaxed tensile modulus in the $<001>$ direction, $E_{<001>}=1 / s_{11}$, is found to be about $29 \%$ too large, while the relaxed shear modulus in the $<111>$ direction, $G_{<111>}=\left[3 c_{44}\left(c_{11}-c_{12}\right)\right] /\left[4 c_{44}+c_{11}-c_{12}\right]$, is about $18 \%$ too large. As a further check on the accuracy of the PAW method, some of the ideal strength calculations have been verified by comparing the energies computed with the FLAPW and PAW methods for structures which were relaxed using the PAW method. These results are described below where applicable.

\section{B. The ideal strength in tension}

First consider the ideal strength in tension. Since previous calculations and symmetry arguments 
suggest $<001>$ is the weakest direction in tension for bcc metals [7], we have focused on the ideal strength in this direction. Figure 1 shows the energy and stress as a function of strain for FM Fe.

\section{Tetragonal vs. orthorhombic instability}

There are two possible deformation paths for uniaxial tension in the $<001>$ direction. An infinitesimal strain in the $<001>$ direction distorts the crystal into a body-centered tetragonal (bct) configuration. If we require that the structure maintain tetragonal symmetry, we find that at $28.6 \%$ strain the energy reaches a local maxima at the fcc structure $(\mathrm{c} / \mathrm{a}$ ratio $=\sqrt{2})$, and then at $42 \%$ strain it reaches a local minima corresponding to a special stress free bct structure with a c/a ratio of 1.66 (see Fig. 2). The plot of stress versus strain associated with this path has a maximum tensile stress of $12.6 \mathrm{GPa}$ at $15 \%$ strain. The maximum locates the elastic instability associated with the bcc-fcc (Bain) deformation path and corresponds, physically, to cleavage on the $\{001\}$ plane.

The initial bct structure can also be treated as a special case of a face centered orthorhombic (fco) structure that is rotated by 45 degrees (see Fig. 2) and has a:b:c ratio of $\sqrt{2}: \sqrt{2}: 1$, where we take the c-axis to lie in the direction of the applied strain. If we preserve orthorhombic symmetry (which allows the tetragonal symmetry to be broken) the deformation is coincident with the Bain path up to a strain of $\sim 18 \%$, but then diverges from it. The evolution of the ratios of the orthorhombic lattice parameters along the loading path is shown at the bottom of Fig. 1 and the geometric relationship between the special crystal structures is shown in Fig. 2. At a strain of $18 \%$ the crystal becomes unstable with respect to an orthorhombic distortion. The energy reaches a maxima at $21 \%$ strain, then falls off into a local minimum at a strain of $42 \%$. The energy maximum along the orthorhombic path occurs at the "special" bct structure with a c/a ratio of 1.66, and the energy minimum is a bcc structure that is rotated relative to the initial bcc structure (Fig. 2). If one refers the structure back to the bct structure with the deformation in the [001] direction, the orthorhombic instability corresponds to the elastic constant $\mathrm{c}_{66}$ vanishing leading the crystal to become unstable with respect to a shear, $\tau_{\mathrm{xy}}$. Although the crystal is pulled in tension this instability would correspond to a shear failure on the $\{211\}<111>$ system of the 
bec crystal.

The competition between tetragonal and orthorhombic instabilities in bcc crystals was first noted by Luo, et al. [19], who found that the tetragonal instability dominates in Mo (as in W) while the orthorhombic instability intrudes in $\mathrm{Nb}$. The issue is physically important, since the tetragonal instability leads to failure in tension ( $\{001\}$ cleavage) while the orthorhombic instability leads to failure in shear $(<111>\{112\}$ slip). Fe behaves like the conventional bcc metals, $\mathrm{W}$ and Mo. Its ideal strength in $<001>$ tension is determined by the tetragonal instability and, hence, by cleavage on $\{001\}$. In keeping with this result, Fe cleaves on $\{001\}$ when tested at low temperature.

The present authors had previously explored the ideal tensile strength of Fe using the FLAPW method, but only considered tetragonal structures. In that work, a slightly higher ideal strength of 14.2GPa was found, which is consistent with the larger energy difference between the bcc FM and fcc FM structures found with the FLAPW method as compared to the PAW method (see Table 1). The energy difference may be due to the differences in the treatment of the GGA or errors introduced by the PAW potential. In the course of that earlier work, the conditions of stability [15] were tested at $14 \%$ strain to check for orthogonal instabilities. No orthogonal instabilities were found at that strain, consistent with the present research. However, the elastic constant $\mathrm{c}_{66}$ was found to be only $\sim 30 \mathrm{GPa}$, quite a bit smaller than in bcc Fe, which was an indication of the close proximity of the orthorhombic instability.

Following Orowon [3], we can model the ideal strength by assuming that the stress strain curve is sinusoidal with an amplitude of $\sigma_{m}$ and a period that is twice the strain needed to reach the fcc phase (2x0.286):

$$
\sigma=\sigma_{m} \sin \left(\frac{e \pi}{0.286}\right) .
$$

Requiring that Hooke's law be satisfied for small strains, one finds that: 


$$
\sigma_{m}=\frac{0.286}{\pi} E_{<100>}=0.091 E_{<100>},
$$

where $E_{<100>}$ is Young's modulus for elongation in the $<001>$ direction. Equation 2 agrees well with the results calculated here: $\sigma_{m} / E_{<100>}=0.087$ (when the experimental value for $E_{<100>}$ is used).

\section{Magnetic instabilities}

The results to this point have only considered ferromagnetic (FM) states; however, the fact that Fe has a metastable, antiferromagnetic fcc phase at low temperature suggests that it must become unstable with respect to magnetic transitions at large strains. We will now consider this possibility.

For computational simplicity we approximate the magnetic structures of fcc $\mathrm{Fe}$ as collinear structures, even though it is fairly well accepted that the ground-state magnetic structures may be complex (such as spin spirals) [36]. It is enormously difficult to treat non-collinear structures. In addition to the problems associated with computing the ground state magnetic structure, one must also ensure that it has the correct stress state. In the computational approach used here we fix the magnetic structure and relax the lattice into the required stress state. We then repeat this process for various magnetic structures. We then increment the strain to obtain the energy and stress as a function of the strain. Increasing the degrees of freedom in the magnetic structure significantly increases the number of magnetic structures that must be tested and structurally relaxed. The inclusion of non-collinear structures dramatically increases the number of magnetic degrees of freedom, and is simply impractical with available computational resources. In addition to assuming collinear structures, we used a scalar relativistic approximation that does not include effects of spin-orbit coupling. However, our earlier calculations using the FLAPW method showed that this makes a negligible effect on the energy differences between various magnetic structures [18].

There is good reason to believe that the collinear approximation, while not exact, may give a 
reasonable representation of the behavior of fcc Fe. There is probative experimental data on the magnetic state of nearly pure Fe in the fcc phase. While bulk fcc Fe is difficult to achieve experimentally at low temperature, it can be stabilized in thin epitaxial films or in small precipitates in a copper matrix. Tsunoda $[37,38]$ found that small fec precipitates in $\mathrm{Cu}$ that are almost pure Fe have a spiral spin density wave ground state. Numerous researchers have studied the magnetic structure of fcc Fe, and Knöpfle et al. [36] have recently published calculations using the modified augmented spherical wave method which show good agreement with experiment. Their minimum energy fcc structure has a spiral vector of $\mathbf{q}=(0.15,0,1)$ with an energy which lies $<1 \mathrm{mRy}$ below the AFM phase and has a slightly larger equilibrium volume than the AFM phase. Herper, Hoffmann, and Entel [39] proposed using a collinear double period anti-ferromagnetic structure (DAFM) as an approximate to the non-collinear ground state. In this structure the spins on (200) planes are oriented up-up-down-down. Their FLAPW results show that the DAFM structure has a similar equilibrium volume and lies $<1 \mathrm{mRy}$ below the AFM phase. This suggests that the DAFM structure may be a good approximate to the non-collinear fcc structure.

In Fig. 3 we present the energy as a function of strain for Fe in the FM, AFM, DAFM and lowspin FM structures. We have studied both bct and fco structures and found that the AFM and LSFM structures remain tetragonal while the DAFM and FM structures undergo a transformation to the fco structure at different points along the loading path. We also see that FM Fe becomes magnetically unstable at about $\sim 20 \%$ strain at which point the DAFM structure is preferred. This result suggests that the ideal strength is not compromised by magnetic instability since the structural instability ( $\{001\}$ cleavage) is encountered before the magnetic instability is reached. This result is in agreement with the earlier FLAPW calculations which only explored the DAFM structure near the fcc structure and near the elastic instability in the FM phase [18]. Thus, the ideal strength in $<001>$ tension is $\sim 12.6 \mathrm{GPa}$ and is set by a tensile elastic instability along the Bain path from bcc to fcc. The stability of fcc iron is made possible by a magnetic phase transition at larger strains which does not affect the ideal strength.

Several previous authors have studied the energy as function of tetragonality for Fe, but have not determined the ideal strength [39, 40]. Friák et al. [41] have calculated the ideal tensile strength 
along the tetragonal loading path using the FLAPW method and found a strength of $12.7 \mathrm{GPa}$ in good agreement with our results from the PAW method and slightly lower than our previous calculations using the FLAPW method. While the use of different GGA formulations in the three cases makes exact comparison difficult, the differences are within the error range for density functional methods. To our knowledge, the only attempt to measure the ideal tensile strength of Fe was by Brenner [42] who tested Fe whiskers in tension. He measured a value of $\sim 5 \mathrm{GPa}$ for tension in the $<001>$ direction [42]. While this value is considerably lower than the calculated ideal strength, the failure initiated at the surface and, therefore, does not represent bulk strength. The measured value of the strength in the $<111>$ direction was $13 \mathrm{GPa}$, but it is expected that the ideal strength of bcc metals in the $<111>$ direction is significantly larger than in the $<001>$ direction $[17,7]$.

\section{The ideal strength in shear}

\section{Shear strength of FM bcc Fe}

We computed the ideal shear strength of Fe for two common slip systems: $<111>\{112\}$ and $<111>\{110\}$. The energy and the stress are plotted as functions of the shear strain for the two systems in Fig. 2. We note that the ideal strength in the easy direction is very similar for both slip systems, 7.2 GPa for $<111>\{112\}$ and $7.8 \mathrm{GPa}$ for $<111>\{110\}$. The energy curves for the two systems also have the same maxima. These results are common in bcc crystals $[9,19]$ and have their origin in the symmetry of the "saddle point" structures that govern the shear strength. Analysis of the saddle point structures for the two slip systems reveals that they are identical. Moreover, the saddle point structure in shear is precisely the bct saddle point structure that governs the orthorhombic instability in tension. The convergence of the three strain paths to the same saddle point simply shows that the nearby saddle points on the energy hypersurface act as "attractors" to which the various deformations are drawn.

While the saddle point structure for the two slip systems is the same, the strain paths on the energy hypersurface are slightly different. This difference is responsible for the fact that the strain at which the bet structure occurs is slightly larger for the $<111>\{110\}$ system, and the 
ideal strengths for the two systems differ by $\sim 8 \%$. The relaxation of the crystal lattice during strain is also distinctly different. A constant volume shear on the $<111>\{112\}$ system (with no relaxation in the slip plane) produces a body-centered orthorhombic structure at a engineering shear strain of $33 \%$. Allowing relaxation, the special bct structure is reached after a strain of $29 \%$ with a $4 \%$ expansion perpendicular to the slip plane, and relaxation strains in the slip plane of $<$ $2 \%$ (see Fig. 5 and Table 2). On the other hand, a $<111>\{110\}$ shear reaches the bct structure at $34 \%$ strain, but requires a significant relaxation in the slip plane, the largest component being a $15 \%$ shear. The reason for the differences between $\{112\}$ and $\{110\}$ shear lies in the fact that while both paths begin at bcc and end at the bct saddle-point structure, the very different applied stresses cause them to diverge from one another at intermediate strains and produce significantly different atomic configurations near the point of instability.

As suggested by Frenkel [2], we model the ideal strength by assuming the that the stress-strain relation is sinusoidal with an amplitude $\tau_{m}$ and period $2 \gamma_{B}$ :

$$
\tau=\tau_{m} \sin \left(\frac{\gamma \pi}{\gamma_{B}}\right)
$$

If we require that Hooke's law be satisfied for small strains, this implies that

$$
\tau_{m}=\frac{\gamma_{B}}{\pi} G_{<111>}
$$

where $G_{<111>}$ is the shear modulus in the $<111>$ direction. (Note that the shear moduli are the same for both shear systems.) If we take $\gamma_{B}$ to be 0.34 , then equation 4 implies that $\tau_{m} / G_{<111>}=0.11$. Our results give dimensionless strengths which are very close to this, for $\{112\} \tau_{m} / G_{<111>}=0.11$ and for $\{110\} \tau_{m} / G_{<111>}=0.12$, where we have used the experimental value of $G_{<111>}$ from Table 1 .

The shear strength in the hard direction for the $<111>\{112\}$ system is $15.0 \mathrm{GPa}$. It follows a path 
toward a different saddle point that is much higher in energy (see Fig. 4 and 5). The saddle point structure is base centered orthorhombic with an a:b:c ratio of $1: 1.06: 1.77$. This structure can also be pictured as a simple tetragonal structure with a c/a ratio of 2.42 which has undergone an (engineering) shear strain of $6 \%$ in the plane perpendicular to the unique axis. Figure 5 demonstrates the geometry and the fact that the strain needed to reach this saddle point is approximately twice that for shear in the easy direction.

To test the numerical accuracy of the PAW method, the energies of several structures along the $<111>\{112\}$ "easy" shear path were also computed with the FLAPW technique. The energy differences were less than $0.2 \mathrm{mRy}$. While we have not confirmed that the stress states of these structures are pure shear when computed with the FLAPW method, the close agreement in the energies along the deformation path suggests that the PAW method is quite accurate in this regime.

\section{Magnetic instabilities in shear}

We also investigated the possibility that finite shear strain would induce magnetic instabilities that would limit the shear strength. This was done by testing several different magnetic structures and relaxing the stresses, as in the ferromagnetic case. The energy was only computed at a few interesting points along the $<111>\{112\}$ loading path. The magnetic structures were chosen to mimic the DAFM structure used for the tensile strength calculations, but because the crystal is monoclinic along the loading path, there are several inequivalent ways of arranging the up and down spins in such a fashion. The 5 structures we studied are shown in Fig. 6. They include superlattices made up of one or two base centered monoclinic cells, with different arrangements of the up and down spins occupying the sites.

Figure 7 shows the energy as a function of strain for the relaxed structures. There is a magnetic transformation at $\sim 20 \%$ strain to the magnetic structure denoted DAFMX. However, this strain is beyond the point of elastic instability, so this transformation does not affect the ideal shear strength. The energy as a function of strain for the DAFMX structure was confirmed by FLAPW calculations using a method similar to that described above. We again note that we have only 
considered here a set of collinear structures which we hope approximate the ground state magnetic structure; however, the introduction of non-collinear magnetic ordering may further reduce the energy of some of these structures.

\section{CONCLUSIONS}

Ab-initio total energy calculations using the PAW method were used to calculate the ideal strength of iron in tension and shear. It is, to our knowledge, the first computation of the ideal shear strength for any ferromagnetic material. The ideal tensile strength in the $<001>$ direction is 12.6 GPa and is associated with an elastic instability at 15\% strain along the Bain path from bcc

to fcc. At this strain the structure is stable with respect to both orthogonal elastic instabilities and magnetic instabilities. However, if it were possible to reach larger strains, both an elastic instability toward a face centered orthorhombic structure would occur and a magnetic instability from the bct FM to a DAFM magnetic structure would be encountered. The ideal shear strengths of the two shear systems, $<111>\{112\}$ and $<111>\{110\}$, are 7.2GPa and 7.8 GPa respectively. The ideal shear strengths are very similar because they are determined by the same body centered tetragonal "saddle point structure". (This bct "saddle-point structure" is also responsible for the orthorhombic instability in tension). Along the $<111>\{112\}$ shear path, a magnetic instability toward a complex magnetic structure is found at $20 \%$ strain. Like the magnetic instabilities in tension, it does not compromise the ideal strength because it occurs at larger strains than the elastic instability in the ferromagnetic phase. Despite its complex magnetic behavior and its metastable fcc phase, the ideal strength of Fe is governed by the same elastic instabilities that are found in other bcc metals.

\section{ACKNOLWLEDGEMENTS}

The authors are grateful to Prof. Marvin L. Cohen and Mr. Weidong Luo, Department of Physics, Univ. of California, Berkeley, for helpful discussions. This work was supported by the Director, Office of Energy Research, Office of Basic Energy Sciences, Materials Sciences Division, US Department of Energy. Computational resources have been provided by the National Energy Research Scientific Computing Center, which is supported by the Office of 
Energy Research of the US Department of Energy. All US Department of Energy support was under contract DE-AC03-76SF00098.

\section{REFERENCES}

[1] Kelly A, Macmillan NH. Strong Solids, third edition. Oxford: Clarendon Press, 1986.

[2] Frenkel J. Z. Physik 1926;7:323.

[3]Orowon E. Rept. Prog. Phys. 1949;12:185.

[4]Paxton AT, Gumbsch P, Methfessel M. Phil. Mag. Lett. 1991;63:267.

[5] Šob M, Wang LG, Vitek V. Comp. Mat. Sci., 1997;8:100.

[6] Šob M, Wang LG, Vitek V. Kovove Materialy, 1998;36:145.

[7] Morris JW Jr., Krenn CR, Roundy D, Cohen, Marvin L. In: Turchi PE, Gonis A, editors.

Phase Transformations and Evolution in Materials. Warrendale (PA): The Minerals, Metals and Materials Soc., 2000. pp.187-207.

[8] Roundy D, Krenn CR, Cohen Marvin L, Morris JW Jr., Phys. Rev. Lett. 1999;82:2713.

[9] Roundy D, Krenn, CR, Cohen Marvin L, Morris JW Jr., Phil. Mag. A 2001;81:1725.

[10] Morris JW Jr., Krenn CR, Roundy D, Cohen ML, Mat. Sci. Eng. A 2001; 309:121.

[11]Morris JW Jr., Guo Z, Krenn CR, Kim YH, ISIJ International 2001;41:599.

[12] Krenn CR, Roundy D, Cohen Marvin L, Chrzan DC, Morris JW Jr., Phys. Rev. B 2002;65:13411.

[13] Hill R, Math. Proc. Camb. Phil. Soc. 1975;77:225.

[14] Hill R, Milstein F. Phys. Rev. B, 1977;15:3087.

[15] Morris JW Jr., Krenn CR, Phil. Mag. A 2000;80:2827.

[16] Wallace DC. Thermodynamics of Crystals. New York: J. Wiley, 1972.

[17] Šob M, Wang LG, Vitek V, Mat. Sci. Eng. A 1997;234-236:1075.

[18] Clatterbuck DM, Chrzan DC, Morris JW Jr. Phil. Mag. Lett. 82;2002:141.

[19] Luo Wiedong, Roundy D, Cohen Marvin L, Morris JW Jr. Phys. Rev. B 2002; 66:094110.

[20] Milstein F, Marschall J. Phil. Mag. A 1988;58:365.

[21] Singh DJ, Pickett WE, Krakauer H. Phys. Rev. B 1991;43:11628.

[22] Blöchl PE, Phys. Rev. B 1994;50:17853.

[23] Kresse G, Furthmüller J. Phys Rev. B. 1996;54:11169. 
[24] Kresse G, Hafner J. Journal Phys. Condens. Matter 1994;6:8245.

[25] Kresse G, Joubert D. Phys. Rev. B 1999:59:1758.

[26] Perdew JP, Zunger A. Phys. Rev. B 1981;23:5048.

[27] Ceperly DM, Alder BJ. Phys. Rev. Lett. 1980;45, 566.

[28] Vosko SH, Wilk L, Nusair M. Can. J. Phys. 1980;58:1200.

[29] Perdew JP, Chevary JA, Vosko SH, Jackson KA, Pederson MR, Singh DJ, and Fiolhais C. Phys. Rev. B 1992;46:6671.

[30] Wang Y, Perdew JP. Phys. Rev. B 1991;44:13298.

[31] Blöchl PE, Jepsen O, Andersen OK. Phys. Rev. B 1994;49:16223.

[32] Methfessel M, Paxton AT. Phys. Rev. B 1989;40:3616.

[33] Perdew JP, Wang Y. Phys. Rev. B 1992;45:13244.

[34] Acet M, Zahres H, Wassermann EF, Pepperhoff W. Phys. Rev. B 1994;49:6012.

[35] Rayne JA, Chandrasekhar BS, Phys. Rev. 1961;122:1714.

[36] Knöpfle K, Sandrastskii LM, Kübler J. Phys. Rev. B 2000;62:5564.

[37] Tsunoda Y. J. Phys. Condens. Matter. 1989;1:10427.

[38] Tsunoda Y, Nishioka Y, Nicklow RM. J. Magn. Mag. Mat. 1996;128;133.

[39] Herper HC, Hoffman E, Entel P. Phys. Rev. B 1999;60:3839.

[40] Stixrude L, Cohen RE, Singh DJ. Phys. Rev. B 1994;50:6442.

[41] Friák M, Šob M, Vitek V. Proceedings of the International Conference Juniormat 2001, Institute of Materials Engineering, Brno University of Technology, Brno 2001, p. 117. [42] Brenner SS. J. Appl.Phys. 1956;27:1484. 
PAW FLAPW PAW-FLAPW Experiment PAW-Experiment

$\%$ Error

bcc-FM

$\mathrm{V}_{\mathrm{o}}\left(\mathrm{au}^{3}\right)$

76.47

76.20

2.827

196

289

152

114

184

78.9

$\mathrm{G}_{<111>}(\mathrm{GPa})$

\section{fcc-AFM}

$\mathrm{V}_{\mathrm{o}}\left(\mathrm{au}^{3}\right)$
$\mathrm{B}(\mathrm{GPa})$
$\mathrm{E}_{\mathrm{c}}(\mathrm{mRy})$

fcc-FM

$\mathrm{V}_{\mathrm{o}}\left(\mathrm{au}^{3}\right)$

80.81

80.70

169

12.0

11.5

$\mathrm{E}_{\mathrm{c}}(\mathrm{mRy})$

160

71.50

209

7.1

$0.4 \%$

78.94

2.86

174

245

139

122

144

65
$\%$ Error

$-3.1 \%$

$-1.1 \%$

$11.5 \%$

$16.9 \%$

$5.9 \%$

$-18.5 \%$

$29.2 \%$

$18.4 \%$

\section{fcc-LSFM}

$\begin{array}{cccc}\mathrm{V}_{\mathrm{o}}\left(\mathrm{au}^{3}\right) & 70.10 & 70.88 & -1.1 \% \\ \mathrm{~B}(\mathrm{GPa}) & 171 & 207 & -17.1 \% \\ \mathrm{E}_{\mathrm{c}}(\mathrm{mRy}) & 10.2 & 9.0 & 13.7 \%\end{array}$

fcc-NM

$\begin{array}{cccc}\mathrm{V}_{\mathrm{o}}\left(\mathrm{au}^{3}\right) & 69.06 & 69.09 & -0.04 \% \\ \mathrm{~B}(\mathrm{GPa}) & 284 & 286 & -0.8 \% \\ \mathrm{E}_{\mathrm{c}}(\mathrm{mRy}) & 10.4 & 10.1 & 3.2 \%\end{array}$

Table 1. Lattice parameters, volumes, relative energies and elastic constants from PAW, FLAPW and experiment. All energies are relative to bcc-FM which is taken as 0. Experimental elastic 
constants at $4 \mathrm{~K}$ are from [35] and the experimental lattice parameter extrapolated to $4 \mathrm{~K}$ is from [34]. 


\begin{tabular}{|c|c|c|c|c|c|}
\hline & \multicolumn{2}{|c|}{ Tension } & \multicolumn{3}{|c|}{ Shear } \\
\hline & $\begin{array}{l}<001> \\
\text { tetragonal }\end{array}$ & $\begin{array}{l}<001> \\
\text { othorhombic }\end{array}$ & $\begin{array}{c}<111>\{112\} \\
\text { (hard) }\end{array}$ & $\begin{array}{c}<112>\{112\} \\
\text { (easy) }\end{array}$ & $<111>\{110\}$ \\
\hline $\bar{\sigma}_{m}, \tau_{m}(\mathrm{GPa})$ & 12.6 & 12.6 & 15.0 & 7.2 & 7.8 \\
\hline Instability & bct & bct & monoclinic & monoclinic & triclinic \\
\hline$e_{x x}$ & -.042 & -.049 & .037 & -.008 & -.002 \\
\hline$e_{y y}$ & -.042 & -.049 & -0.26 & -.006 & -.001 \\
\hline$e_{z z}$ & .150 & .176 & .042 & .028 & .029 \\
\hline$\gamma_{y z}$ & & & -.374 & .140 & .059 \\
\hline$\gamma_{x z}$ & & & & & .020 \\
\hline$\gamma_{x y}$ & & & & & .035 \\
\hline Saddle Point & fcc & bct & orthorhombic & bct & bet \\
\hline$e_{x x}$ & -.091 & -.070 & .046 & .004 & -.014 \\
\hline$e_{y y}$ & -.091 & -.070 & -.031 & -.016 & -.015 \\
\hline$e_{z z}$ & .286 & .209 & .088 & .044 & .033 \\
\hline$\gamma_{y z}$ & & & -.726 & .288 & .339 \\
\hline$\gamma_{x z}$ & & & & & .022 \\
\hline$\gamma_{x y}$ & & & & & .154 \\
\hline
\end{tabular}

Table 2. Summary of stresses and strains associated with instabilities and saddle points. For tension, the strains are refered to a bct cell with the unique [001] axis along the z-axis. For $<111>\{112\}$ shear, the reference structure is a base centered monoclinic cell with its 2 -fold axis parallel to the $\mathrm{x}$-axis, one orthogonal lattice vector parallel to the the shear direction which coincides with the y-axis, and the other orthogonal lattice vector in the y-z plane. For $<111>\{110\}$ shear, the reference structure is a triclinic cell with one lattice vector in the shear direction which coincides with the y-axis, the second lattice vector in the shear plane which coincides with the $\mathrm{x}-\mathrm{y}$ plane, and third vector with some component in the z-direction. 
(a)

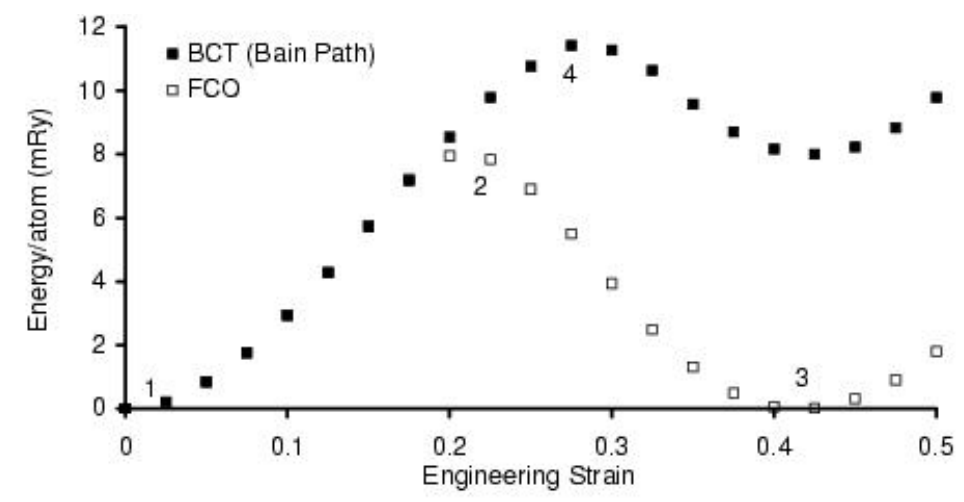

(b)

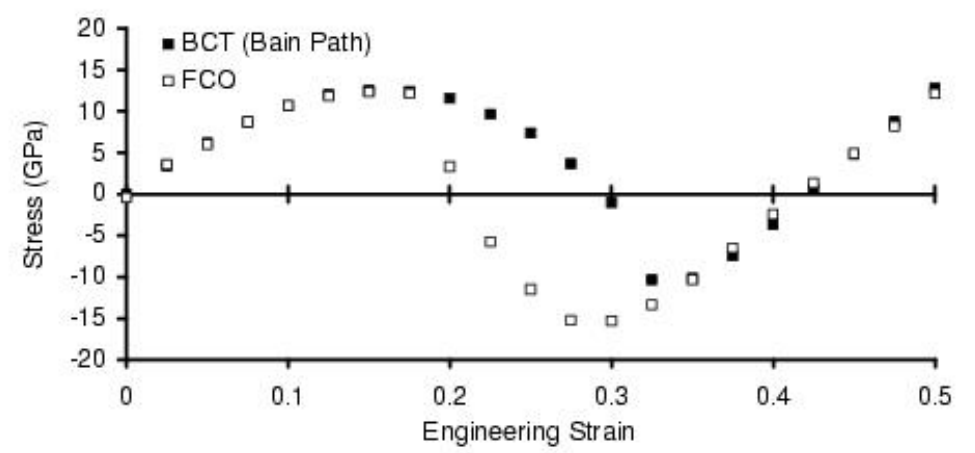

(c)

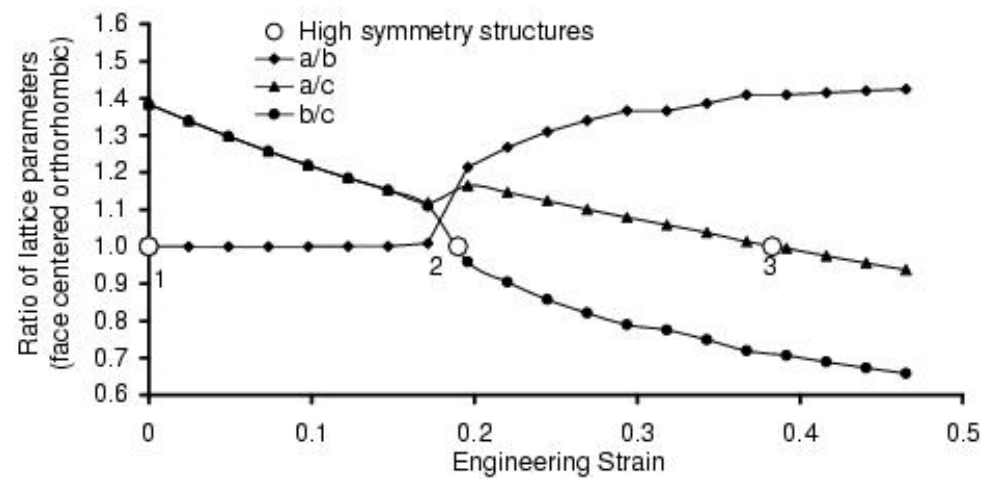

Figure 1. (a) Energy and (b) stress as a function of applied tensile strain along $<001>$ : (filled square $\mathbf{~})$ Bain Path with tetragonal symmetry, (open square $\square$ ) orthorhombic path. (c) Ratios of the lattice parameters along the orthorhombic path: (filled diamond $\diamond$ ) a/b, (filled triangle $\mathbf{\Delta}$ ) $\mathrm{a} / \mathrm{c}$, (filled circle $-\mathrm{O}$ ) b/c. Numbers and open circles in (a) and (c) indicate special structures with high symmetry that are shown schematically in Fig. 2. 


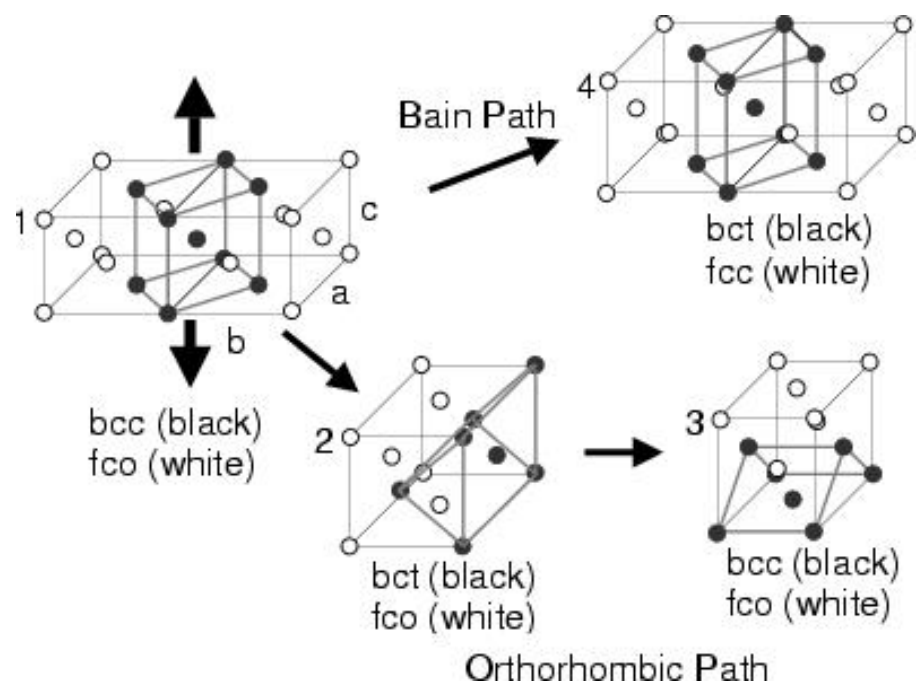

Figure 2. The geometry of the Bain path and the orthorhombic instability. The crystal starts as a bcc structure (1) which can also be visualized as a fco structure with a:b:c ratio of $\sqrt{2}: \sqrt{2}: 1$. If tetragonal symmetry is maintained the Bain path is followed to the fcc structure (4) which is equivalent to a bct structure with a c/a ratio of $\sqrt{2}$. If the tetragonal symmetry is broken the orthorhombic path is followed and a maximum energy is reached at a special bct structure (2) which has a c/a ratio of 1.66. From that energy maxima, the orthorhombic path leads down in energy to a bcc structure (3) which is rotated relative to the starting bcc structure.

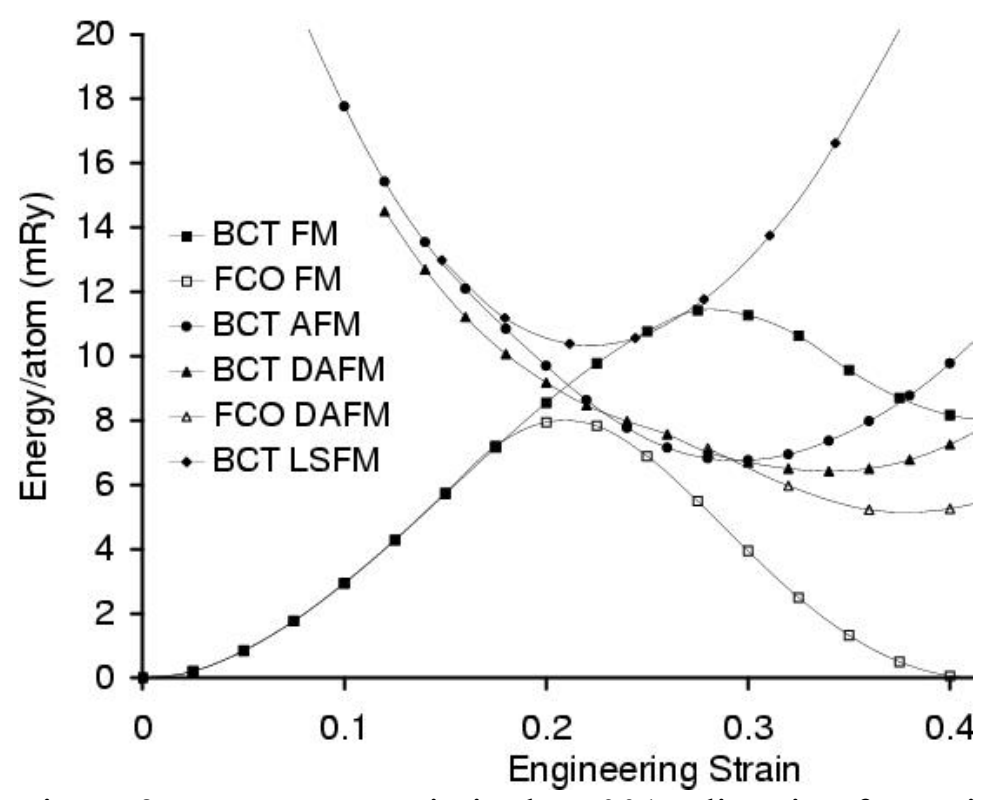

Figure 3. Energy vs. strain in the $<001>$ direction for various magnetic structures: (filled square -) bct-FM, (open square $\square$ ) fco-FM, (filled triangle $\Delta$ ) bct-DAFM, (open triangle $\triangle$ ) fcoDAFM, (filled circle $\bullet$ ) bct-AFM, (filled diamond $\bullet$ ) bct-LSFM. The fco-AFM and fco-LSFM structures follow paths identical to bct-AFM and bct-LSFM respectively and are not shown. A magnetic phase transformation is seen near $20 \%$ strain. Lines are guides for the eye. 
(a)

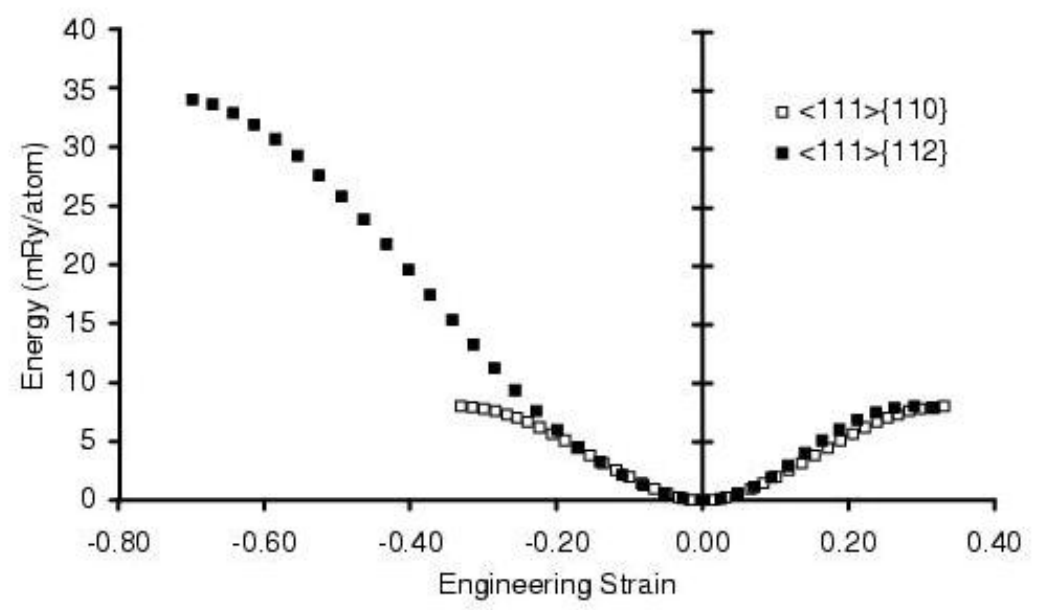

(b)

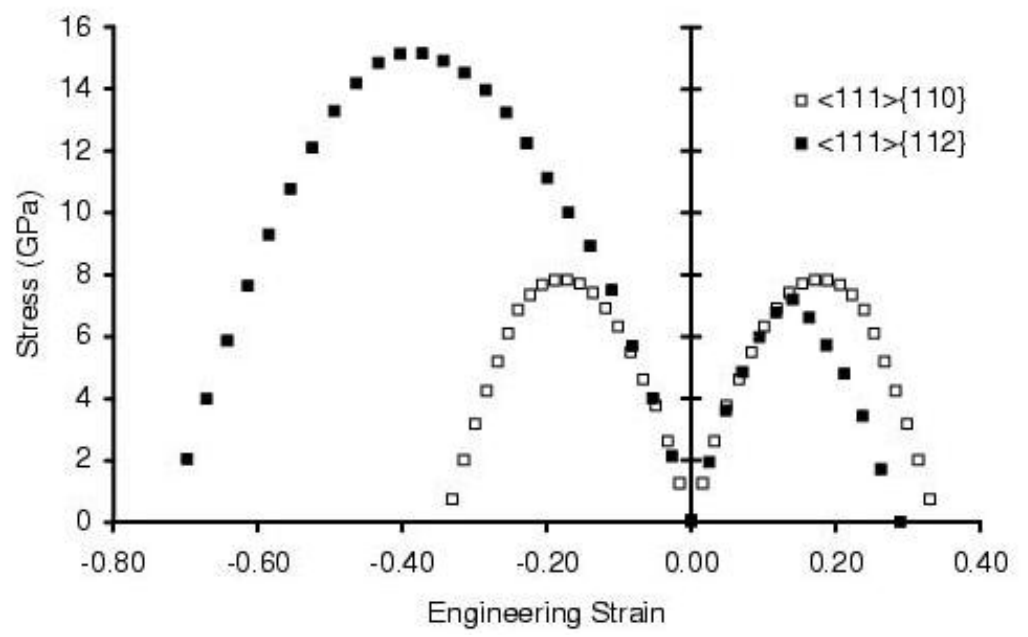

Figure 4. (a) Energy versus shear strain and (b) absolute value of the stress versus strain for (filled square $\mathbf{a})<111>\{112\}$ shear and (open square $\square$ ) $<111>\{110\}$ shear.

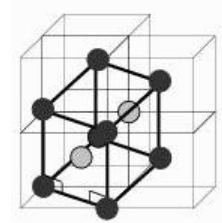

Base Centered Monoclinic Cell
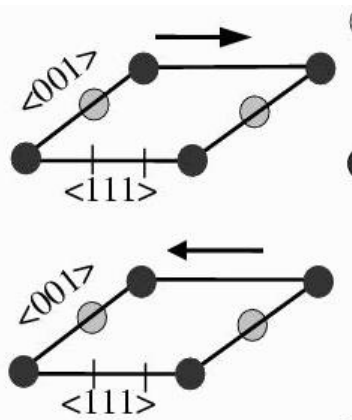

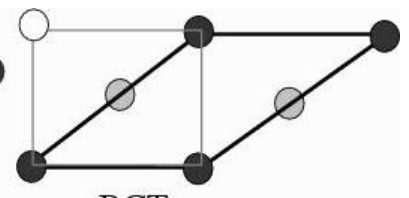

$\mathrm{BCT}$

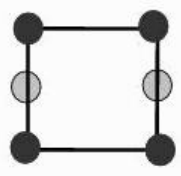

Base Centered

Orthorhombic

Figure 5. Geometry of the $<111>\{112\}$ shear system. The base centered monoclinic cell is shown inside the initial bcc structure (left). A $\{110\}$ projection of the structure shows the how the monoclinc cell becomes a bet cell upon applying a $\sim 33 \%$ shear in the easy direction (top). A shear in the opposite (hard) direction generates a base centered orthorhombic structure after a shear strain of $\sim 66 \%$ (bottom). The monoclinic structure's two fold axis is pointed out of the page. Black atoms are those in the closest plane and the gray atoms are in the following plane of atoms. 


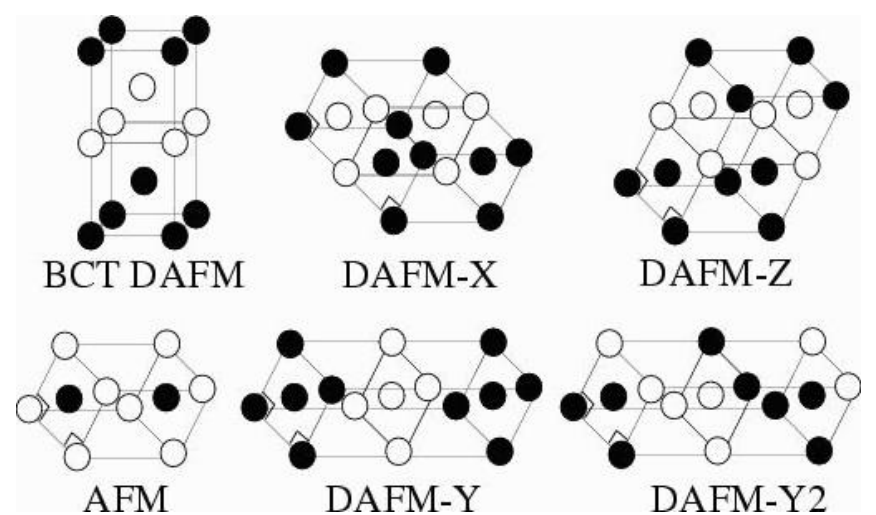

Figure 6. The magnetic structures used to describe complex magnetic ordering in $<111>\{112\}$ shear. The structures are supercells made up of 1 or 2 body centered monoclinic cells whose 2fold axes are oriented out of the page. The bct-DAFM structure is shown for reference.

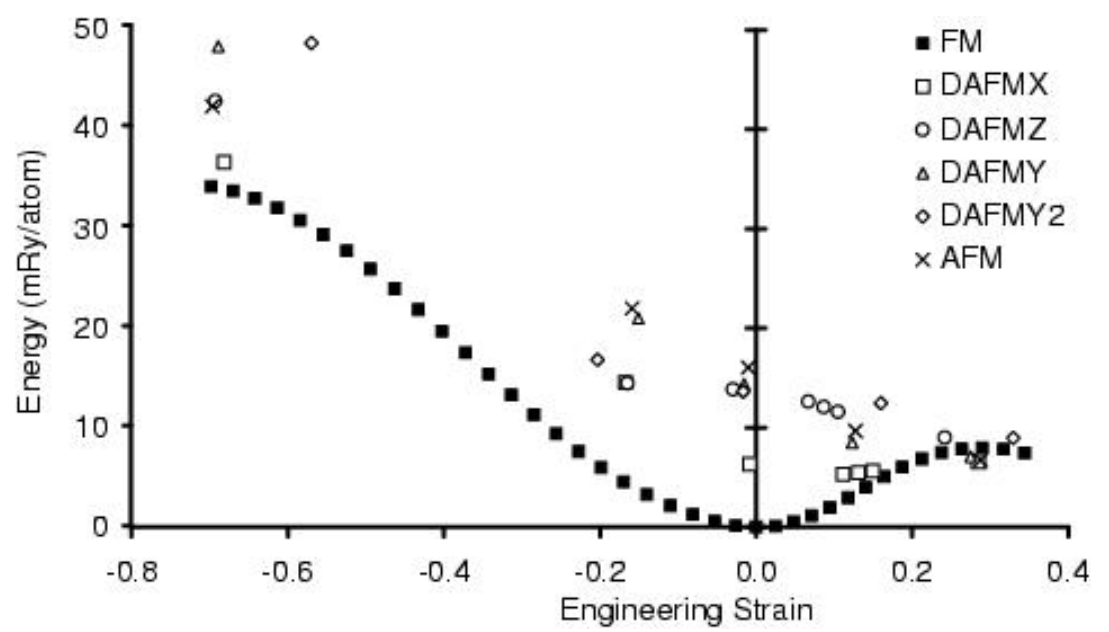

Figure 7. Energy as a function of shear strain for several magnetic structures. (solid square a) FM, (open square $\square$ ) DAFMX, (open circle O) DAFMZ, (open triangle $\triangle$ ) DAFMY, (open diamond $\diamond)$ DAFMY2, (X) AFM. The various magnetic structures are shown in Fig. 6. 